\title{
Progress in Domain-Engineered Photonics Materials
}

\author{
Yalin Lu, ${ }^{1}$ Hiroshi Murata, ${ }^{2}$ and Chang-Qing $\mathrm{Xu}^{3}$ \\ ${ }^{1}$ Department of Physics, United States Air Force Academy, Colorado Springs, CO 80840, USA \\ ${ }^{2}$ Division of Advanced Electronics and Optical Science, Department of Systems Innovation, \\ Graduate School of Engineering Science, Osaka University, 1-3 Machikaneyama, Toyonaka, Osaka 560-8531, Japan \\ ${ }^{3}$ Department of Engineering Physics, McMaster University, Hamilton, ON, Canada L8S 4L7
}

Correspondence should be addressed to Yalin Lu, yalin.lu@usafa.edu

Received 9 November 2008; Accepted 9 November 2008

Copyright (C) 2008 Yalin Lu et al. This is an open access article distributed under the Creative Commons Attribution License, which permits unrestricted use, distribution, and reproduction in any medium, provided the original work is properly cited.

Artificially engineering ferroelectric and ferromagnetic oxide materials' domain structures provide many potential opportunities to explore such materials' extraordinary nonlinear optic, electrooptic (EO), and magnetooptic (MO) effects, to build unique photonic bandgap structures, and to generate phonon-photon-coupled polaritons. Domain engineering can be applied onto such materials, either one-dimensinally or two-dimensionally, can be patterned on the above materials periodically, quasiperiodically, or aperiodically, or can be aligned along different crystalline orientations or by using complicated cascaded structures. Those commonly involved photonic materials can be versatile including ferroelectricferromagnetic oxide crystals, semicondcutors, electrooptic polymers, and so on, in either bulk, thin film, or waveguide forms. Implementation of such domain engineering can be very versatile too, and it may follow direct crystal growth, superlattice growth, overgrowth on an already patterned structure, electrical field poling, E-beam writing, and so on. Potential applications of such domain-engineered materials for photonics are very widespread, including nonlinear frequency conversion, EO modulation, optical bistability, acoustics, ultrasonic transducers, terahertz $(\mathrm{THz})$ generation, fiber optics, left-hand materials, and so on.

A remarkable example of past researches in this area is the realization of the quasi-phase-matched (QPM) nonlinear frequency conversions using periodically poled crystals. Recently, development of new photonic and optoelectronic components using such advanced domain-engineered materials, covering a broad range of operation frequencies and having unique optical functions, has become very attractive. However, the effort toward this direction has been crucially relying on the availability of new optical materials, new physical mechanisms, and new device designs. In this special issue focusing on exploring such new aspects, we invited a few papers that address the major issues in the area, summarized some of those recent progresses, and discussed those emerging opportunities of applications.

The first two papers of this special issue are related to the realization of such domain-engineered structures. The first article from M. Fujimura and T. Suhara is the specially invited one, which reports a new formation method for making domain-inverted gratings in $\mathrm{MgO}: \mathrm{LiNbO}_{3}$ crystal at room temperature via applying an electric field to the crystal under the irradiation of UV light. The results support the unique way to use a photoconductive cladding layer to suppress the excessive lateral expansion of the domaininverted regions. The formation process does not require the use of photolithography processing and allows a full room temperature operation. Therefore, it is simple and productive. The second article describes the fabrication of proton-exchanged (PE) waveguides on domain-inverted stoichiometric $\mathrm{LiTaO}_{3}$ (SLT) crystals for guided-wave EO modulation applications. The extraordinary index change in SLT via PE with a coefficient of $0.25 \times 10^{-12} \mathrm{~cm}^{2} / \mathrm{s}$ was found to be 0.017 , which is comparable to that from the congruent $\mathrm{LiTaO}_{3}$ crystal. Guided-wave EO modulation using such waveguides was also demonstrated.

The following four papers are discussing various issues in a few different nonlinear frequency conversion processes including second harmonic generation (SHG) and difference frequency generation (DFG). Among them is the fifth article by Y. Wong et al. which is also a specially invited one. The third paper by $\mathrm{S}$. Chu et al. reports an $\sim 1 \mathrm{~W}$ continueouswave green light generation in a bulk periodically poled 
$\mathrm{MgO}: \mathrm{LiNbO}_{3}$ crystal, using the intracavity QPM design andexcited by a diode-pumped $\mathrm{Nd}: \mathrm{YO}_{4}$ laser. The paper that follows actually describes the SHG in an MgO-doped periodically poled congruent $\mathrm{LiNbO}_{3}$ crystal and pumped by an efficient all-fiber Q-switched Yb-doped fiber laser which delivers high-output power and long-pulse width. The conversion efficiency reaches $4.2 \%$ that agrees well with the theoretical simulation. The specially invited article by Y. Wang et al. touches a very unique side in the SHG process by studying the noise characteristics of both harmonic and fundamental waves at relatively higher-power levels, through analyzing their time-domain and frequency-domain characteristics. Understanding the noise characteristics has strong impacts on many applications including coherent detection, spectroscopy, free-space telecommunication, and so on. The fourth paper in this group expands the scope of the interest to detect an optical signal by transferring the signal from the optical frequencies to microwave frequencies via the second-order susceptibility-based DFG process. This frequency-transition method not only offers the potential of a major detection efficiency improvement, but also works well for both intensity-modulated and frequency/phasemodulated optical signals. The study impact to improve the modulation bandwidth in optical fiber telecommunication will be deep.

The seventh and eighth articles in this special issue are directly focusing on EO modulators made from domainengineered ferroelectric crystals. The seventh paper by H. V. Pham et al. discusses a new method to design traveling-wave EO modulators with fully controlled frequency responses, using nonperiodically domain-reversed structures. Frequency responses of both magnitude and phase of modulation index will be artificially controllable using such new nonperiodical designs. In this paper, several EO modulators for advanced modulation formats such as duobinary modulation and wideband single-sideband modulation are proposed. The eighth article actually discusses the estimation of the phase velocity of a modulation microwave in a quasi-velocity-matched (QVM) EO phase modulator, using the unique EO sampling method that should be very accurate and the most reliable for measuring voltage waveforms on modulator electrodes.

Moving forward from the above discussions on frequency conversion and EO modulation, the last two papers of this special issue actually touch the terahertz $(\mathrm{THz})$ wave generation in a specially designed nonlinear optical fiber, and, even further, the realization of negative optical refraction in a multilayered structure that modulated both tunable dielectric and magnetic behaviors. For avoiding the common absorption problem in current nonlinear optical materials, a multicladding fiber design having a periodically poled $\mathrm{LiNbO}_{3}$ fiber as the maincore was proposed. The generated $\mathrm{THz}$ waves via DFG will instantly be coupled to the outer cladding made from those polymeric materials having low absorption over a broad range of $\mathrm{THz}$ frequencies, and the optical beam will be maintained inside the main $\mathrm{LiNbO}_{3}$ fiber core. The last article proposes a new theory of realizing negative refraction by frequency-tuning to concurrence of both dielectric layer and the magnetic layer inside a multilayered structure. Negative refractive index will appear after the concurrence frequency. This theory is significant, since the anticipated negative index metamaterials will be flexible in fabrication, and have strong impacts on emerging areas such as superlens, optical cloaking, and sensing.

Yalin $\mathrm{Lu}$

Hiroshi Murata

Chang-Qing Xu 

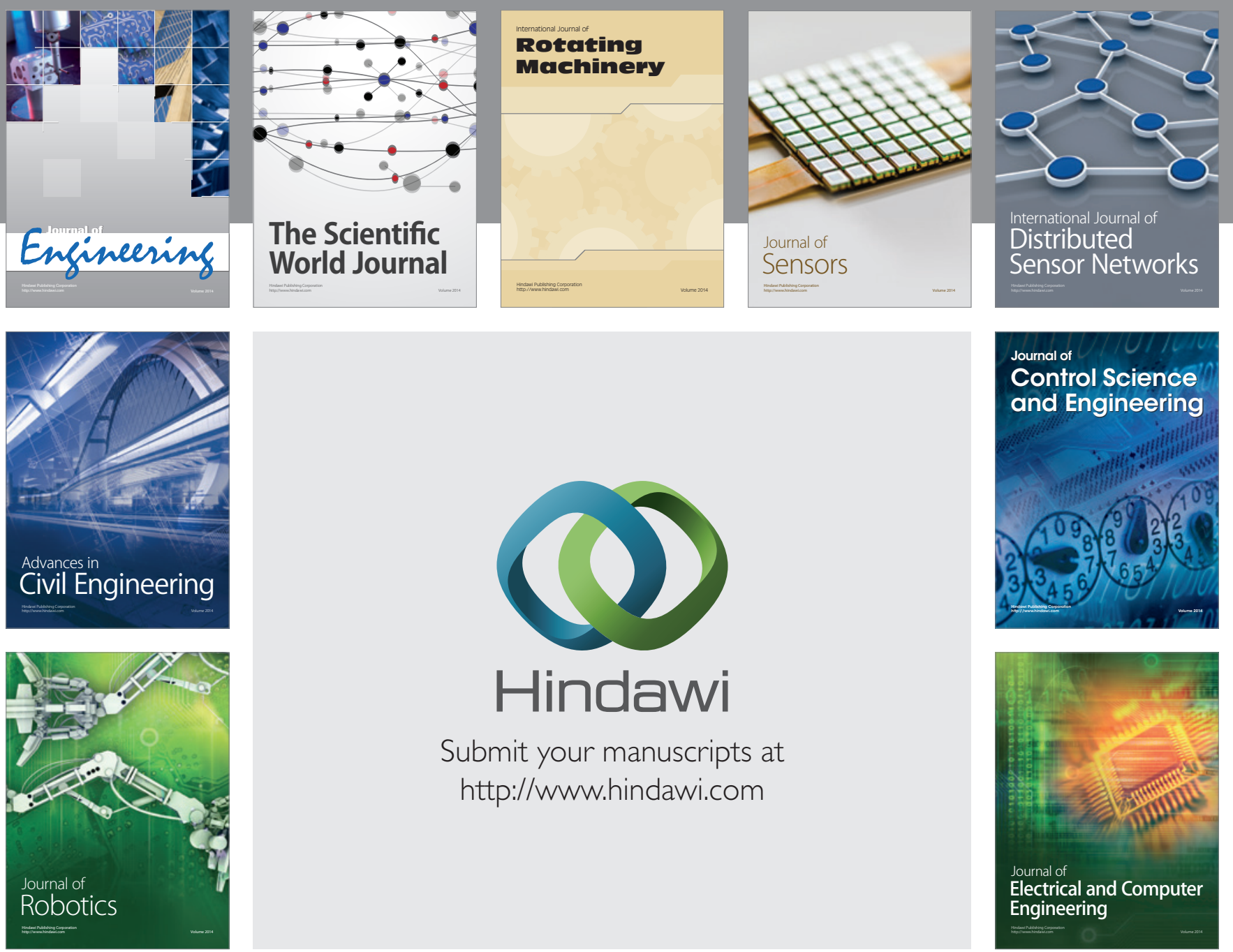

Submit your manuscripts at

http://www.hindawi.com
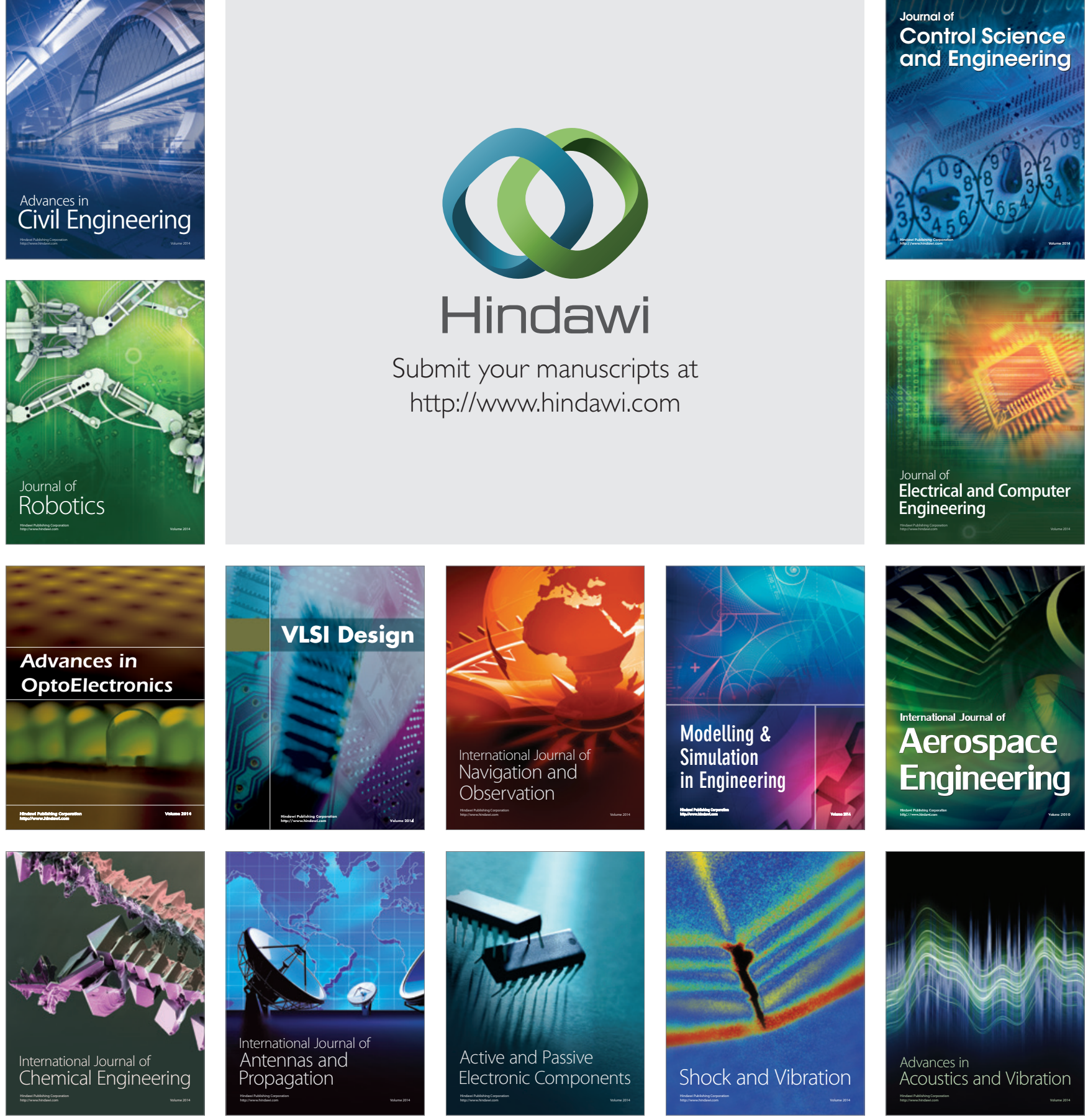